$J J M L L$

\title{
Entre Ici et Là-Bas, Vit un Petit Pays
}

\author{
Rana Alameen* \\ Institut français, Jordanie
}

Received on: 9-2-2020

Accepted on: 16-8-2020

Résumé

Petit Pays de Gaël Faye est l'histoire d'un enfant et d'une patrie. Gaby vit au Burundi dans un confortable quartier d'expatriés. Il est entouré de ses amis, de sa famille et d'un bonheur quil croyait éternel. Ce quotidien paisible, cette enfance douce vont se disloquer en même temps que ce pays d'Afrique brutalement malmené par l'histoire. La guerre bouleverse son pays, sa famille, son quartier et même son rêve d'être 'mécanicien'. Notre présent article vise à suivre l'évolution d'un thème qui évoque des conditions de vie très différentes entre 'ici' et 'là-bas'. Cet enfant né à Bujumbura et exilé en France est le héros qui nous emporte vers les coins 'chauds' et 'froids' de son existence. La relation entre ses deux vies est pour nous une rencontre particulière et l'occasion d'une réflexion sur la notion d' 'habitat'.

Mots-clés: Burundi, Enfance, Violence, Séparation, Exil, Patrie.

\section{Between Here and There, Lives a Small Country}

\begin{abstract}
Petit Pays by Gaël Faye is the story of a child and a homeland. Gaby lives in Burundi in a comfortable expat neighborhood. He is surrounded by his friends, his family and a happiness that he believed to be eternal. This peaceful daily life and this sweet childhood will fall apart at the same time as this African country is brutally battered by history. The war upsets his country, his family, his neighborhood and his dream of being a 'mechanic' disappears. Our present study aims to follow the evolution of the theme which evokes very different living conditions between 'here' and 'there'. This child, born in Bujumbura and exiled in France, is the hero who takes us to the 'warm' and 'cold' corners of his existence. The relationship between his two lives is for us a special encounter and an opportunity for a reflection on the notion of 'habitat'.
\end{abstract}

Keywords: Burundi, Childhood, Violence, Separation, Exile, Homeland.

\section{Introduction}

"La guerre entre les Tutsi et les Hutu, c'est parce qu'ils n'ont pas le même territoire? Pas le même pays? Pas la même langue? Pas le même Dieu?" "- Non, ce n'est pas pour ça "répond le père. "Alors pourquoi se font-ils la guerre?" reprend Gabriel. - "Parce qu'ils n'ont pas le même nez!" dit le père (Faye 2016, 10).

Nourri d'un drame que l'auteur connaît bien (Alix 2016, 147), le premier roman de Gaël Faye a fait partie du dernier carré d'ouvrages sélectionnés par l'Académie Goncourt pour son prix 2016. Cet auteur

\footnotetext{
๑ 2021 JJMLL Publishers/Yarmouk University. All Rights Reserved.

* Doi: https://doi.org/10.47012/jjmll.13.2.3

* Corresponding Author: amin.ranou@yahoo.com
} 
franco- rwandais compositeur et interprète de rap est aussi influencé par la littérature créole que par la culture hip-hop. Son Petit Pays remporte le prix Goncourt des lycéens 2016 et a été également sélectionné pour d'autres prix: prix du premier roman 2016, prix du roman Fnac et bien d'autres (La Meselée 2016, 2).

Gaël Faye ne représente pas le génocide, ni la guerre civile: "la littérature ne pourrait pas décrire une telle horreur" dit-il (Fruchon-Toussaint 2016). L'auteur évoque les tourments et les interrogations d'un enfant pris dans une histoire qui le fait grandir plus vite que prévu. Ce sont ces questions qui se mêlent avec une centaine d'images tantôt douces d'une couleur gaie, remplies de parfums de manguiers et de citronnelles, d'une pluie et d'un soleil chauds; tantôt sombres où la peur, le sang, la guerre, linsécurité surgissent et métamorphosent le petit Gabriel en un être qui ne peut plus rester enfant, qui ne peut plus rester neutre à l'égard de cette guerre ; un enfant qui ne veut plus devenir 'mécanicien' car selon lui il n'y a rien à réparer, plus rien à sauver et plus rien à comprendre.

Né au Burundi d'une élégante mère rwandaise et d'un père français, Gabriel vit à Bujumbura avec son père sa mère et sa petite sœur Ana dans un confortable quartier d'expatriés. Un quotidien paisible, une enfance douce vont se disloquer en même temps que ce petit pays d'Afrique brutalement malmené par l'Histoire (Sow 2011, 15).

"Comme tout récit défini comme une quête" (Reuter 1991, 51), le héros de Gaël Faye se met tout au long du roman à la recherche d'un pays, d'une famille, d'un 'soi' perdus dans les milles détails d'une guerre ethnique incompréhensible (Ngorwanubusa 2014,127). Mais cette tâche n'est certainement pas la seule. La véritable mission de cet enfant est de défier l'oubli et la mort avec une âme fragile et puissante à la fois! Ainsi l'enfant nous invite à prendre conscience de la barbarie humaine se mêlant à son imagination: "Certains soirs le bruit des armes se confondait avec le chant des oiseaux." (Faye 2016, 180).

On a toujours dit que la vie est un roman et ce n'est sûrement pas l'auteur du Petit Pays qui dirait le contraire; lui qui essaye à travers 'Gaby' de sauver ce qui reste d'une patrie par le prisme de l'écriture. "Le bruit de la télévision couvre un instant le cours de ma pensée. Une chaîne d'infos en continu diffuse des images d'êtres humains fuyant la guerre. J'observe leurs embarcations...Les enfants qui en sortent sont transis de froid. L'opinion publique pensera qu'ils ont fui l'enfer pour trouver l'Eldorado... On ne dira rien du pays en eux." (Faye 2016, 16) Par-là, nous pouvons déjà repérer les indices de ce pays intériorisé que l'émigrant Gabriel transporte en lui.

Nous suivrons alors dans ce présent article l'évolution de la notion 'habitat' dont le sens ne consiste plus à occuper un lieu; mais à associer le vécu et l'imaginaire. Cette notion fait l'objet d'une analyse visant à mieux saisir, comprendre et distinguer les différentes parties du roman.

Dans La première partie du livre consacrée au bonheur d'un quotidien ordinaire, 'Le Burundi' est capable d'assurer à 'Gaby' la joie et la sécurité (Sow 2011, 12). Ce 'chez soi' disparaît dans La seconde partie du roman et marque la fin du bonheur, de linnocence et de la famille. Dans cet horizon qui s'obscurcit, les murmures des adultes laissent deviner que la folie des hommes est en marche. La guerre éclate, le sang coule et l'enfance n'existe plus. Gaby s'éloigne de la beauté des lacs, de la douceur des collines et des manguiers pour chercher un abri quelque part à Paris, là où il ne retrouve plus son 'chez soi' . 
Dans la première étape de l'analyse de la notion en question, la définition du verbe "Habiter" nous parait fondamentale. Les différents sens du verbe non-pas contradictoires mais complémentaires deviennent la quête même d'un équilibre personnel du héros. Et après ces longues années de pluie et de 'nulle part', Gaby décide de rentrer, c'est le grand départ vers la patrie. Mais, entre un tel 'exil' et un tel 'retour' retrouvet-il la même chaleur de son petit pays? C'est à cette question que nous allons tenter de répondre dans la partie 'Exil et retour'. Il est intéressant aussi de consacrer une petite partie de ce présent article à Gaby en cherchant la manière à travers laquelle il réussit à se définir et à ressentir sa place et son rôle dans cette histoire.

\subsection{Habiter}

L'homme a toujours eu besoin de s'abriter, de se protéger et de s'approprier des espaces. De la philosophie à la géographie, de l'anthropologie à l'architecture, de la sociologie à l'histoire, la notion d'habitat traverse les sciences sociales et humaines. 'Habiter': est un verbe polysémique, un verbe qui s'emploie au transitif comme à l'intransitif, tout en changeant subtilement de sens. D'après le Petit Robert, le premier synonyme du mot habiter est 'vivre'. Il est principalement question de logement, de demeure, d'un endroit où on réside. Un autre sens, qui recouvre les sens précédents, mais plus symboliquement, est celui qui fait intervenir l'existence humaine. Habiter un espace, c'est remplir cet espace de soi-même, y laisser son empreinte. C'est donc la relation entre l'homme et sa demeure qui permet d'habiter. Pris dans un sens plus large que se loger, 'habiter' engage ainsi tout un rapport au monde (Courtés 2003, 15).

Habiter est au fondement même de la nature de l'homme. Dès que le petit d'homme quitte le ventre maternel, il est projeté dans un autre espace; il est accueilli dans un lieu qui va le fabriquer et évoluer avec lui. La demeure de l'enfance est au point de départ, la première expérience d'un autre monde, d'une construction de soi. Tout homme a quitté la maison de l'enfance, mais pour beaucoup la maison de l'enfance ne les a jamais quittés.

L'auteur du Petit Pays élabore à partir de l'expérience de son personnage sa propre interprétation d'habiter'. Les étapes qui marquent le sens de ce mot correspondent à la construction de l'identité. L'auteur y revient plusieurs fois pour souligner ce qui constitue le noyau de l'expérience d'habiter, comment l'histoire personnelle de son personnage raconte son 'espace habité' et comment les modes de vie deviennent l'expression d'une personnalité.

Jusqu'à l'âge de onze ans Gaby habitait au Burundi. Ce lieu délimité qui différencie son espace privé du reste du monde: "Au temps d'avant, avant tout ça [...] C'était le bonheur, la vie sans se l'expliquer. L'existence était telle qu'elle était, telle qu'elle avait toujours été et que je voulais qu'elle reste. Un doux sommeil, paisible, sans moustique qui vient danser l'oreille." (Faye 2016, 19) Le Burundi apparaît comme le nid, la mémoire heureuse de l'origine avec ses qualités de repos, de tranquillité et de sécurité.

'Sa' maison d'enfance est un milieu d'expériences avec des murs, des portes, des fenêtres, un sol, des bruits, des parfums, des coins chauds et des amis de quartier: "on passait notre temps à se disputer avec les copains, mais y a pas à dire, on s'aimait comme des frères. Les après- midi, on filait tous les cinq vers notre quartier, on discutait, on décidait nos projets, nos escapades, nos grandes vadrouilles. On rêvait beaucoup, 
on s'imaginait, le cœur impatient, les joies et les aventures que nous réservait la vie. En résumé, on était tranquilles et heureux..." (Faye 2016, 74) Mais que s'est-il passé après ?Où ont disparu les coins chauds de la maison, le sol, les bruits, les amis, le quartier et le Bonheur?

Parti en France à cause de la guerre civile au Burundi, Gaël Faye nous transporte dans une toute autre condition d'existence et sur un autre sens du verbe 'habiter': "Je n'habite plus nulle part. Habiter signifie se fondre charnellement dans la topographie d'un lieu, l'anfractuosité de l'environnement. Ici (en France) rien de tout ça. Ma cité est dortoir et fonctionnelle. Mon appartement sent la peinture fraiche et le linoleum neuf. Mes voisins sont de parfaits inconnus, on s'évite cordialement dans la cage d'escalier." (Faye 2016, 13).

Comment expliquer 'je n'habite nulle part'? Comment habiter dans un terme indiquant justement l'absence du lieu? Est-ce un endroit isolé ou une situation incertaine? Ce 'nulle part' permet en fait à l'auteur d'orienter son combat contre la mort et l'oubli. Cette incertitude de l'espace empêche Gaby de se représenter à lui-même et d'avoir part à l'espace qui lui permet de s'identifier. 'Le nulle part' de Gaby c'est un espacetemps arrêté qui lui permet de revenir à son passé, aux lieux physiques quill porte en lui. Cette technique 'd'espace-temps arrêté' donne l'occasion à Gaël Faye de présenter son héros comme un chercheur qui se livre à un travail d'analyse entre ses propres lieux et les lieux où il habite. Cet 'espace- temps' immobile devient un lieu d'échange entre ce 'nulle part' et celui de Gaby. Pour lui, 'Habiter' se fait par émotion et non plus par raison. Une telle signification soulève une autre question: quel est donc le rapport de l'être humain avec l'espace où il habite, lorsque le seul espace habitable est l'âme?

La demeure est un corps d'images qui apporte à l'homme une certaine stabilité. C'est un endroit qui nous accueille, un 'bleu' plus soutenu que 'le ciel'. La fonction première de notre maison est de nous abriter. Mais inconsciemment, c'est aussi et surtout un prolongement de notre moi et de notre corps. On est 'chez soi' lorsque le lieu ne peut être celui d'autrui, on possède son propre espace lorsque l'on peut s'y retirer pour s'isoler du reste du monde. En France Gabriel n'a pas trouvé les espaces de 'chez soi', il n'a pas trouvé son petit coin de l'univers et cela explique bien "le nulle part" du paragraphe precedent.

En France, c'est sa demeure, son Burundi, son 'chez soi', ses souvenirs d'enfance, ses instants joyeux qui vivront désormais dans l'espace de son corps et de son âme. Il essaye ainsi de trouver 'la sérénité intérieure' nécessaire pour affronter la vie et ses frustrations. Ce sentiment d'être à l'abri de tout danger ou risque: "La nuit, me revient le parfum de mes rues d'enfance, le rythme calme des après- midi, le bruit rassurant de la pluie qui tambourine le toit de tôle. J'observe le marronnier effeuillé dans le square en bas de mon immeuble. J'imagine à sa place la puissante voûte des manguiers qui rafraichissait mon quartier." (Faye 2016, 212) Ici, les images sont des opérations qui produisent un écart et une dissemblance (Rancière 2003 , 10). Le pays est décevant pour Gaby parce qu'il ne correspond pas aux images dont il est fortement empreint. Cet enfant d'Afrique n'est plus sous le soleil chaud de son pays et les orages de Paris pénètrent son cœur.

Pendant vingt ans, le corps et l'âme de Gabriel constituent une référence et un endroit où habite le territoire de l'intimité; le Burundi : "pas un jour sans que le pays ne se rappelle à moi. Un bruit furtif, une odeur diffuse, une lumière d'après- midi, un geste, un silence parfois..." (Faye 2016, 13) C'est par la 
préservation de l'intimité que l'habitat tient un rôle important de préservation de soi. Mais Le problème pour Gaby réside dans le degré de ressemblance entre l'image du moment réel et les photographies d'autrefois.

Il y a donc, selon l'auteur du Petit Pays, 'l'habitat' avec domicile celui 'du Burundi' et 'l'habitat' de l'homme exclu, sans domicile celui 'de la France', "Je tangue entre deux rives, des milliers de kilomètres me séparent de ma vie d'autrefois. Ce n'est pas la distance terrestre qui rend le voyage long, mais le temps qui s'est écoulé. J'étais d'un lieu, entouré de famille, d'amis, de connaissance et de chaleur." (Faye 2016, 213). Cette image est d'autant plus significative qu'elle ne cesse de se répéter en empruntant chaque fois le même procédé qui consiste à saisir le passé 'sur le vif'.

Jusqu'à l'âge de trente ans, Gaby constitue autour de soi un territoire exclusivement personnel. La recherche d'un chez soi se montre essentielle et continue. Mais avec tous ses efforts de trouver cette 'sécurité'; il est bien difficile pour lui de se mettre à distance du pays qui habite son âme. Il ressent le froid dans l'âme ainsi que le froid Celsius. Il veut changer d'air parce que ce dernier est irrespirable et tout ce que ses yeux voient est inconnu: "Je viens de si loin que je suis encore étonné d'être là ... je m'approche de la fenêtre. Le ciel est bas. Il pleut... il n'y a aucun manguier dans le petit parc coincé entre le centre commercial et les lignes de chemin de fer." (Faye 2016, 15).

Gabriel a cherché désespérément à faire partie intégrante de ce tableau de la vie en France. Il semble aussi que l'espace de l'âme ne suffise plus pour la préservation de son pays. Il est temps pour lui de se "fondre charnellement dans la topographie d'un lieu" (Faye 2016, 13) pour y habiter. Ce cri témoigne d'un amour exigent envers l'Afrique et appelle à la compréhension de soi-même. Il prend sa décision ; "Je dois y retourner". (Faye 2016, 16).

\subsection{Exil et Retour}

Deux anniversaires renvoient à deux moments, deux lieux, deux vies très différents. L'un marque la fin du bonheur et l'autre marque le retour. A onze ans, à Bujumbura, il fête son anniversaire entouré de 'ceux qu'il aimait': "Ce soir-là, je savourais le Bonheur...Papa respirait la gaieté et la bonne humeur...Quant à Maman, elle resplendissait, dans sa robe fleurie en mousseline..." (Faye 2016, 104) Gaby célébrait l'existence, l'instant et l'éternité de ses onze ans. Vingt ans plus tard, seul en France, il court se réfugier dans le premier bar et commande un whisky pour fêter ses trente-trois ans." (Faye 2016, 16) Entre ces deux espaces, ses "onze ans" et son "whisky", il existe un terrain d'expériences qui illustre parfaitement les coins 'chauds' et 'froids' de son existence.

"La guerre à Bujumbura s'était intensifiée. Le nombre de victimes était devenu si important que la situation au Burundi faisait désormais la une de l'actualité internationale. Un ministre envoyé par Paris est arrivé à Bujumbura avec deux avions de rapatriement pour les ressortissants français. Papa nous a inscrits pour le départ. Une famille d'accueil nous attendait, Ana et moi, là-bas, quelque part en France, à neuf heures d'avion de notre impasse." (Faye 2016, 210). Cette ombre, cette guerre ethnique, ce détachement arrachent Gaby à sa patrie, au milieu où il est né et où il a grandi et à soi-même.

Gabriel vivra ainsi un double exil: son exil personnel loin de ses parents presque divorcés et restés à Bujumbura et la séparation brusque avec le Burundi, l'enfance et la joie. Il suffit de lire ces lignes pour 
constater cette double souffrance: "Quand on quitte un endroit, on prend le temps de dire au revoir aux gens, aux choses et aux lieux qu'on a aimés. Je n'ai pas quitté le pays je l'ai fui. J'ai laissé la porte grande ouverte derrière moi et je suis parti..." (Faye 2016, 211).

Gaby se trouve à un moment très particulier de son histoire. Fuir n'est pas son choix, il y est forcé en raison de cette violence qui règne chez lui. Il Laisse derrière lui un pays qui n'a pas su offrir des rêves à ses enfants. Fuir pour lui c'est perdre une grande partie de ses repères, sa ville, ses amis et sa culture. Mais pour survivre et échapper à la disparition de tout cela, prenons un exemple qui montre comment l'auteur confronte cette menace : "Au moment de lui dire au revoir (à sa voisine), elle a filé vers sa bibliothèque et a déchiré une page d'un de ses livres. C'était un poème... Elle m'a dit de garder ces mots en souvenir d'elle..." (Faye 2016, 210). Ces mots sont certainement les repères de sa racine et de son Burundi. La puissance de ce 'poème' réside sans doute dans le lien entre son enfance et son avenir.

Ce départ, ces brefs moments de panique, cet adieu, cet abandon, ce vide dessinent l'image d'une rupture qui l'envahit et creuse une profonde blessure. Tout seul sous l'effet de ce choc Gaby se souvient "simplement de la petite main de papa qui s'agitait au balcon de l'aéroport de Bujumbura". (Faye 2016, 211) La dernière voix qu'il avait entendue avant son départ n'était pas celle de sa mère qui était "quelque part à Bujumbura", mais celle de sa voisine: "Prends garde au froid, veille sur tes jardins secrets, n'oublie jamais d'où tu viens..." (Faye 2016, 211). Une telle déclaration illustre parfaitement lidée de 'la fin du bonheur', d'une nouvelle aventure, de nouveaux horizons qui l'attendent. Loin de son enfance, Gaby grandit dans une ambiance tout à fait différente.

L'auteur du Petit Pays ne dit presque rien de la vie douloureuse de son personnage en France. Il consacre seulement quelques pages à ces années d'exil. On rencontre après vingt ans de séparation et de fuite Gabriel disant: "ma vie ressemble à une longue divagation... Rien ne me passionne... Je m'observe en société, au travail, avec mes collègues de bureau. Est-ce bien moi, ce type? Ce garçon près de la machine à café qui se force à rire? Je respire mal. J'élargis le col de ma chemise. J'observe mes chaussures cirées, elles brillent, me renvoient un reflet décevant. Que sont devenus mes pieds? Ils se cachent. Je ne les ai plus jamais vus se promener l'air libre." (Faye 2016, 15) Le narrateur est incapable d'accepter cet individu qui n'est pas lui. Ce 'reflet' est certainement 'décevant' parce qu'il ne renvoie pas sa véritable image.

Pendant ces longues années d'éloignement non désirées, il ne cherche pas une vie meilleure, il veut juste survivre, il ne fait "que passer. Loger. Crécher. Squatter" (Faye 2016, 13) Il a l'impression d'être seul. Il n'a pas retrouvé en ces vingt ans la chaleur humaine ayant le grand pouvoir sur son bonheur: "... une lourde mélancolie s'abat sur moi, comme une pluie tropicale quand je repense à Papa, Maman, les copains..." (Faye 2016, 16).

Cette mélancolie resurgit surtout quand il regarde "des images d'êtres humains fuyant la guerre." (Faye 2016, 16) Ces images réveillent des angoisses d'enfance notamment chez celui qui a subit avant vingt ans la même séparation, la même fuite et la même solitude. Dans cette même nuit, dans ce même bar et devant ces mêmes images, le héros décide de reprendre le chemin vers son pays et de se mettre à la recherche de son passé et des traces de son enfance. Selon lui "Si l'on est d'un pays, si l'on y est né, on l'a dans les yeux, la peau, les mains, avec la chevelure de ses arbres, la chair de sa terre, les os de ses pierres, le sang de ses 
rivières, son ciel, sa saveur, ses hommes et femmes..." (Faye 2016, 213) Cette description possède un double objet: d'une part révéler ce qui unit l'homme à sa nation et ses racines, d'autre part comprendre la difficulté de s'intégrer quand on vient de si loin.

Dans cet ailleurs, Gabriel retrouve surtout l'envie d'arranger les choses, de trouver des solutions. Ce long voyage est l'occasion de revenir plus serein, mieux dans ses 'chaussures', prêt à gérer les choses différemment et à faire des efforts pour retrouver son Burundi: " Ne serait-ce que pour en avoir le cœur net. Solder une bonne fois pour toute cette histoire qui me hante. Refermer la porte derrière moi, pour toujours." (Faye 2016, 16).

Le retour, c'est la période la plus délicate après ce long voyage. Gabriel désire reprendre sa vie d'avant, comme s'il était seulement parti quelques semaines en vacances. Il voulait retrouver ses proches, ses amis et le confort de sa vie d'enfance. Il voulait peut-être décrire ses aventures à tous ses amis. Le retour pour lui est l'occasion de retrouver après autant de temps passé son bonheur perdu. Il imagine peut-être que s'il était resté, les choses, les gens et l'âme du lieu n'auraient pas disparu. Mais rien de tout ça.

Arrivé au Burundi, il a retrouvé l'endroit "mais il est vide de ceux qui le peuplaient, qui lui donnaient vie, corps et chair." (Faye 2016,213) A l'âge de trente et un ans Gabriel n'a plus reconnu ce pays qui vivait en lui. Le pays n'est plus le même: "j'ai retrouvé l'impasse. Vingt ans plus tard. Elle a changé. Les grands arbres du quartier ont été rasés. Le soleil écrase les journées. Des murs de parpaings surmontés de tessons de bouteilles et de fil barbelé ont remplacé les haies colorées de bougainvilliers. L'impasse n'est plus qu'un morne couloir poussiéreux, ses habitants des anonymes confinés..." (Faye 2016, 213).

A Bujumbura, "une voix d'outre-tombe, me pénètre les os... Je bouscule des ombres, trébuche contre des casiers de bières, tâtonne dans le noir, m'approche du fond de la cabane... Je la retrouve vingt ans plus tard, qui ont compté cinquante sur son corps méconnaissable. Je me penche vers la vieille dame. J'ai l'impression qu'elle me reconnait, à la façon dont elle me fixe à la lueur du briquet que j'approche de son visage. Avec une tendresse infinie, Maman pose délicatement sa main sur ma joue..." (Faye 2016, 216).

On peut alors avancer à travers ces deux citations que les représentants du 'Bonheur' d'autrefois forment la toile de fond d'un réel qu'il n'a pas souhaité voir. L'état de la mère et du Burundi de Gaby est décevant parce quill ne correspond plus aux images de son enfance. Le choc provoqué par cette réalité ne l'arrête pas, il veut à tout prix la couleur gaie de son passé.

Ainsi, la rencontre de Gaby avec son pays et sa mère, cette unité qui vient redoubler l'enveloppe maternelle, déclenche chez lui une nouvelle vitalité. Il admet que les deux ont bien changé, les deux ne vont pas bien et décide alors de "rester ici" (Faye 2016, 217 (et attendre qu'elles aillent mieux toutes les deux: sa mère et sa patrie. Il a la sensation d'être le seul à vraiment comprendre ce qu'ils ont vécu: "Le jour se lève et j'ai envie de l'écrire." (Faye 2016, 217) dit-il à la dernière page de son roman.

\subsection{Un enfant invincible}


Le choix d'un narrateur de dix ans permet à l'auteur d'introduire une dimension poétique à son œuvre. La montée de la violence et les conflits ne sont pas analysés du point de vue d'un adulte mais par celui d'un enfant pour qui le cercle amical et familial est plus important que la vie politique (Brinker 2014,310- 326). Ce combattant réussit à se définir à travers tout ce qui peut menacer son innocence: la séparation de ses parents, la violence et la fin de la paix dans son 'impasse'.

Gaby connaît les joies d'une enfance libre et heureuse "comme au premier jour d'un coup de foudre." (Faye 2016, 154) Entouré de sa bande de copains, il se sent très puissant. À cette époque "ils étaient les nouveaux rois de la rue." (Faye 2016, 71) Limpasse, le lac et la voiture Volkswagen encadrent la zone de sécurité pour ces enfants: "On discutait, on rigolait, on fumait des Supermatch en cachette, on écoutait des histoires incroyables et des blagues...." (Faye 2016, 73).

Mais la limite entre le monde des enfants et le monde des adultes disparaît brusquement quand cet enfant prend conscience de la violence qui s'approche de lui. Gaby voit avec inquiétude ses parents se séparer, puis la guerre civile se profiler, suivie du drame rwandais. La fin de ce bonheur s'annonce quand le jeune garçon assiste simultanément aux disputes de ses parents et aux prémices d'une guerre civile qui oppose Hutus et Tutsis: "Papa ne voulait pas qu'on en parle, pour lui, les enfants ne devaient pas se mêler de politique. Mais on n'a pas pu faire autrement". (Faye 2016, 10).

La guerre prive 'Gaby' de son entourage protecteur, elle le transforme complètement; il devient plus fragile, plus inquiet et plus 'adulte'. La peur parvient peu à peu à instaurer une vie différente. "Je ne me souviens plus de ce que nous avons fait durant cette période. Je ne me souviens ni de l'école, ni des copains, ni de notre quotidien. A la maison...un immense trou noir nous a engloutis, nous et notre mémoire..." (Faye 2016, 163) En effet; Gaby est trop jeune pour comprendre ce qui se passe. Il essaye de développer par son imagination et son courage un moyen de se défendre face au danger et garder un peu de poésie au sein de son foyer loin des hommes en feu: " Ce soir-là, avant d'aller au lit, j'ai emprunté une lampe torche dans un des tiroirs du secrétaire de papa. Sous les draps, j'ai commencé à lire le roman... Au fil de la lecture, mon lit se transformait en bateau..." (Faye 2016, 169).

Mais la violence à Bujumbura "avait pour conséquence de faire pousser grillages, vigiles, alarmes et barrière..." (Faye 2016, 172) Le père de notre héros a pris sa décision: "Il nous a inscrits, Anna et moi pour le départ en France" (Faye 2016, 210) Dans ce cadre tout à fait différent, l'auteur développe une stratégie basée sur le concept de résistance non violente. Pendant ces longues années d'exil, la résistance psychologique de Gaby se traduit par les images et les souvenirs d'enfance. Mais L'éloignement de son pays est terrible, au point de n'avoir d'autre choix que de repartir. A l'âge de trente- trois ans, ce jeune homme suit le "signe du destin." (Faye 2016, 16). C'est donc le temps de ce voyage après de tant d'années d'absence, de distance, d'indifférence et de détachement. C'est le temps de toucher sa terre natale de ses propres mains.

Gaël Faye choisit un enfant qu'il dote de dons et de talents pour une mission spécifique. Il sent sert comme d'un canal par lequel il manifeste sa puissance en vue d'accomplir son œuvre. Cet enfant du monde est la voix de toutes les personnes réfugiées, "une voix qui parle juste, et qui parle à tous" (Gary 2016). Gaby est un véritable lutteur. Il traduit à travers ses forces, sa faiblesse, son enfance et sa maturité l'espoir de surmonter tous les obstacles et les échecs d'une vie qui semblaient irréparables. L'auteur présente un 
héros ordinaire pour une mission extraordinaire: faire revivre un pays perdu; "je retrouve un peu de ce Burundi éternel que je croyais disparu." (Faye 2016, 215).

\section{Conclusion}

C'est autour de la blessure que s'articulent de nouvelles modalités de l'expression. A la réalité immobile des pays perdus dans la barbarie des guerres, répond une écriture mobile qui s'achemine dans tous les sens et devient l'espace de ces terres errant dans nos cœurs. Ainsi, L'acte d'écrire va de pair avec l'acte de vivre (Rancière 2000, 13-14). L'écriture semble être la seule stratégie pour échapper à l'impossible et à l'oubli. Dans ce roman le Petit Pays de Gaël Faye est situé entre deux vies; celle de l'exil avec toutes ses douleurs et celle du retour qui était plus cruel encore: "Je pensais être exilé de mon pays. En revenant sur les traces de mon passé, j'ai compris que je l'étais de mon enfance." (Faye 2016, 213) La tentative de l'auteur incarne non seulement son désir de reproduire et recréer la réalité, mais aussi le désir de défier et de vaincre la mort du Bonheur.

Les lieux évoqués dans ce roman qui respire le vécu, existent dans plusieurs réalités. Ces lieux physiques que l'on porte en nous, transmis par nos mots et nos émotions, ces lieux qui nous habitent, qui nous appellent de très loin pour toucher le territoire de l'intimité et trouver les détails d'une vie et d'un pays perdus; révèlent l'histoire d'une nostalgie bien connue de tous ceux qui ont vécu le bonheur englouti dans une guerre éprouvante et sanglante.

Mais cet appel peut se réaliser seulement à travers l'écriture car on ne pourra jamais retrouver le bonheur, l'enfance et la chaleur de ces 'lieux' démolis et écrasés par les guerres. Ainsi, écrire sera nonseulement pour le futur mais encore pour le présent; sorte de miroir qui aide à se reconnaître, à sidentifier,

à se retrouver: "La poésie n'est pas de l'information, pourtant, c'est la seule chose qu'un être humain retiendra de son passage sur terre." (Faye 2016, 16).

On peut donc déduire que le Petit Pays de Gaël Faye participe à une véritable communication ' hors temps'. De l'histoire d'un enfant à celle du monde, de la quête individuelle à la quête universelle; on touche le secret de l'avenir: la littérature devient petit à petit l'éternité de nos pays, nos soleils et nos joies disparus.

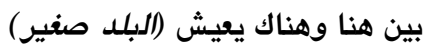




$$
\begin{gathered}
\text { المركز الثقافي الفرنسي، الأردن } \\
\text { الأمين }
\end{gathered}
$$

تناولت رواية (البلد الصغير) للكاتب غايل فاي قصة طفل وبلد في آن واحد. يعيش غابي في حي مريح للمغتربين في بوروندي فهو محاط بعائلته وأصدقائه وحياة سعيدة كان يعتقد أنها أبدية. وهكذا تمضي سطور هذه الرواية لتعبر عن تحول حياة هذا الطفل التي سوف تنهار في نفس الوقت الذي يدمر فيه بلده بوحشية. سحقت الحرب بلده وأسرته وجواره وحتى حلمه بأن يكون "ميكانيكياً . تهدف دراستنا الحالية إلى متابعة تطور مصطلح "المسكن" الذي تطرق الكاتب من خلاله إلى ظروف معيشية مختلفة للغاية بين "هنا" و "هناك". هذا الطفل المولود في بوجومبورا والمنفى في فرنسا هو البطل الذي يأخذنا إلى

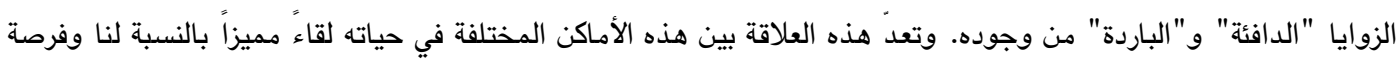
للتأمل والتعليق. الكلمات المفتاحية: بوروندي، الطفولة ، العنف، الانفصال، المهجر، الوطن.

\section{Références}


Bouchard, Louise. 1985. Les images. Les Herbes Rouges. Montréal.

Brinker, Virgine.2014. La Transmission littéraire et cinématographique du génocide des Tutsis au Rwanda. Classiques, Garnier. Paris.

Chrétien, Jean-Pierre. 2000. L'Afrique des grands lacs. Deux mille ans d'histoire. Flammarion. Paris.

Courtés, Joseph. 2003. La sémiotique du langage. Nathan. Paris.

Faye, Gaël. 2016. Petit Pays. Grasset. Paris.

Florian, Alix. 2016. Gaël Faye, Petit Pays. Dans Afrique contemporaine 2016/1, vol. 257.

Fruchon-Toussaint, Catherine. «Petit Pays n'est absolument pas mon histoire », entretien avec Gaël Faye, RFI, mis en ligne le 9 septembre 2016, http://www.rfi.fr/culture/20160908-gael-faye-petit-paysabsolument-pas-histoire.

Gary, Nicolas. Le roman de Gaël Faye, Petit Pays, sollicité par les éditeurs du monde entier sur ActuaLitté, le 28 août 2016.

La Meselée Marin, Valérie. Gaël Faye, chouchou des prix littéraires sur Le Point, le 1er septembre 2016.

Ngorwanubusa, Junéval.2014. Le Regard étranger. L’image du Burundi dans les littératures belge et française. Peter Lang. Bruxelles .

Rancière, Jacques.2000. Le Partage du sensible. Esthétique et politique. La Fabrique éditions. Paris.

Rancière, Jacques. 2003. Le destin des images. La Fabrique éditions. Paris.

Reuter, Yves.1991. Introduction à l'analyse du roman. Bordas. Paris.

Ricœur, Paul. 1990. Soi-même comme un autre. Seuil, col. "L'ordre philosophique". Paris.

Sow, Alioune. 2011. Vestiges et vertiges. Récits d'enfance dans les littératures africaines. Artois Presses Université. Arras. 